\title{
Combination of zerumbone and cisplatin to treat cervical intraepithelial neoplasia in female BALB/c mice.
}

\begin{abstract}
Recent in vitro and in vivo studies have demonstrated that zerumbone (ZER) possesses anticancer properties. The main objective of this study was to examine the effectiveness of the combination of ZER and cisplatin (CIS) to treat cervical intraepithelial neoplasia (CIN) in vivo. Microculture tetrazolium assay and immunohistochemistry of proliferating cellular nuclear antigen were used to study the antitumor effect of ZER.Prenatally exposed female $\mathrm{BALB} / \mathrm{c}$ micewere used as a model. The progenies with CIN were injected peritoneally with isotonic sodium chloride solution (positive control), CIS, ZER, and a combination of both compounds. All treated and untreated mice were humanely killed, and serum and cervix were obtained for interleukin 6 analysis and histopathologic studies using hematoxylin and eosin staining, respectively. Zerumbone has revealed an antitumor effect on human cervical cancer cells and downregulates immunoexpression of proliferating cellular nuclear antigen $(\mathrm{P}<$ 0.05). In vivo study indicates that ZER at $16 \mathrm{mg} / \mathrm{kg}$ and CIS at $10 \mathrm{mg} / \mathrm{kg}$ have a regressing effect on CIN. The combination of ZER and CIS also showed similar effectiveness in regressing CIN. Our results indicate that the combination of ZER and CIS has modulated the serum level of interleukin 6 when compared with that in mice treated with isotonic sodium chloride solution $(\mathrm{P}<0.05)$. The effectiveness of combining ZER and CIS could be further explored as a new therapeutic intervention of early precancerous stages of carcinogenesis before the invasive stage begins.
\end{abstract}

Keyword: Cervical intraepithelial neoplasia; Cisplatin; Synergy; Zerumbone. 